\title{
CONSTRUCTION AND ANALYSIS OF LONG-TERM SURFACE TEMPERATURE DATASET IN FUJIAN PROVINCE
}

\author{
W. E. $\mathrm{Li}^{\text {a }}{ }^{*}, \mathrm{X}$. Q. Wang ${ }^{\mathrm{a}}, \mathrm{H} . \mathrm{Su}^{\mathrm{a}}$ \\ ${ }^{a}$ Key Laboratory of Spatial Data Mining \& Information Sharing of Ministry of Education, National Engineering Research Centre of \\ Geo-spatial Information Technology, Fuzhou University, China1836682015@qq.com
}

\author{
Commission IV, WG IV/3
}

KEY WORDS: MODIS, Land surface temperature, Spatio-temporal variation, Remote sensing, Fujian Province

\begin{abstract}
:
Land surface temperature (LST) is a key parameter of land surface physical processes on global and regional scales, linking the heat fluxes and interactions between the ground and atmosphere. Based on MODIS 8-day LST products (MOD11A2) from the splitwindow algorithms, we constructed and obtained the monthly and annual LST dataset of Fujian Province from 2000 to 2015. Then, we analyzed the monthly and yearly time series LST data and further investigated the LST distribution and its evolution features. The average LST of Fujian Province reached the highest in July, while the lowest in January. The monthly and annual LST time series present a significantly periodic features (annual and interannual) from 2000 to 2015. The spatial distribution showed that the LST in North and West was lower than South and East in Fujian Province. With the rapid development and urbanization of the coastal area in Fujian Province, the LST in coastal urban region was significantly higher than that in mountainous rural region. The LST distributions might affected by the climate, topography and land cover types. The spatio-temporal distribution characteristics of LST could provide good references for the agricultural layout and environment monitoring in Fujian Province.
\end{abstract}

\section{INTRUDUCTION}

\subsection{General Instructions}

Land surface temperature (LST) is a crucial variable in global and regional climate changes, hydrological cycles, and surfaceatmosphere interactions (Ermida et al., 2017). Characterizing changes in LST is increasingly important for understanding the present environmental conditions and predicting or managing future changes (Pan et al., 2007). The construction of LST timeseries dataset can reflect the law of continuous change of surface temperature, which is of great significance for the monitoring of dynamic changes of surface coverings in the study area (Qin R., 2013; Quan et al., 2016). In general, the construction of LST long sequence data set plays significant role in studying terrestrial ecosystem process and global change. In addition, the distribution characteristics and the level of LST also provide valuable references for rationally arranging agriculture and preventing cold damage. Meanwhile, taking use of meteorological observation data to study the distribution of the LST, which has difficulty in fully reflecting the spatial distribution of surface temperature due to complex terrain and other factors. In order to overcome such limitations, the use of remote sensing data inversion LST is undoubtedly a feasible and useful fashion, which has macro, real-time, dynamic continuous characteristics for continuously monitoring and studying a large area of surface temperature.

In recent years, the use of MODIS data to carry out the study of surface temperature has been progress, showing better than the previous NOAA AVHRR spatial resolution and inversion accuracy(Pan et al., 2007). Zhan et al. (2011) attempted to MODIS data of July to August from 2001 to 2007 in coastal region of Fujian province of land surface temperature (LST) and
NDVI, to monitor annual changes and evaluate the UHI effect. Wang et al. (2012) analyzed the temporal and spatial distribution characteristics of Land Surface Temperature (LST) in Shaanxi province using MODIS/LST time series data from 2001 to 2011. Guan et al. (2015) researched the characteristics of land surface temperature (LST) of Tianshan Mountain area based on the MODIS /MOD11A2 data from 2001 to 2013 in 2015. As previous studies showed above, research of long time series LST datasets mostly construct in the northern inland city, less for coastal cities due to lack of data.

The first objective of this paper is to establish a quantitative long LST time series data in Fujian province. Long-term sequence of LST derive from monthly-scale and yearly-scale to illustrate the variation trend of the LST within the study domain. The second objective is to dynamically monitor and analyze the spatial distribution characteristics of LST in Fujian province from 2000 to 2015.

\section{STUDY AREA}

Fujian Province is located in the southeast coast of China,

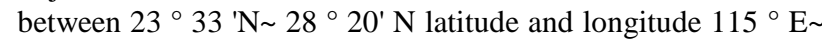
$120^{\circ} 40^{\prime} \mathrm{E}$. It has a typical south subtropical monsoon climate in the southern coastal areas and a middle subtropical climate in the northern mountainous and hilly areas. The annual temperature of $15.3 \sim 21.9^{\circ} \mathrm{C}$. It has abundant rainfall, rich in calories, forest coverage ranked first in the country (forests cover $68.7 \%$ of Fujian Province). However, it as a mountainous and frequent resource disasters province, agricultural production is largely affected by typhoons, rainstorm and other natural

factors. 


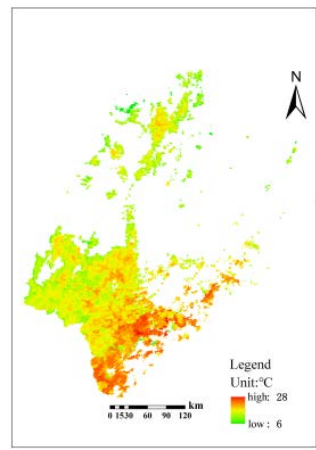

(a) 313 days

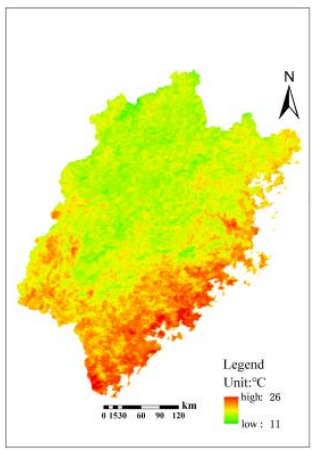

(b) 321 days

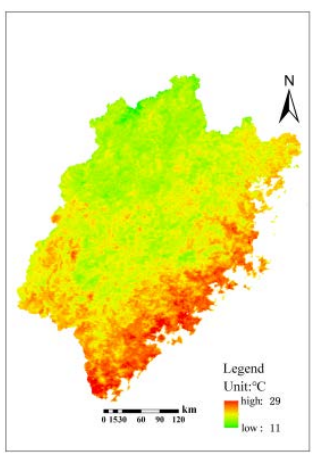

(c) 329 days

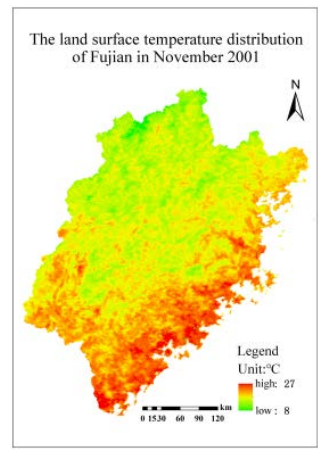

(d) November 2001

Figure 1. The 8-days LST products in the days of 313, 321 and 329 on November 2001 in Fujian Province (a, b, c) and the monthly synthetic LST map based on the three images (d)

\section{DATA AND METHOD}

\subsection{Basic Data}

The study used 8-day time series of 1000m MODIS land surface temperature data (MOD11A2), acquired from March 2000 to 2015 (http://e4ftl01.cr.usgs.gov/MOLT/MOD11A2.005/); The annual mean temperature data and larger almanac of climate change in Fujian Province from 2000 to 2015, etc.

\subsection{Data Processing}

MOD11A2 has 12 scientific datasets, we should remove the abnormal pixels by the quality control file (QA band). Firstly, we need to batch process the coordinate conversion and reprojection; Then, we can clip the boundary, based on the interest region (Fujian Province), converse temperature unit and other basic data procession and so on. But the most critical question is synthesizing. The Fujian province is so cloudy and rainy all the year round that it difficult to collect better quality MODIS images (especially in summer). For MODIS images, artificially discarded more missing parts of image (such as: cloud cover). But for sporadic scrawled countless area, we used the interpolation method with neighboring data to fill it. Every MODIS image is cloud-detected and accordingly marked. Based on the non-cloud pixels in the image of each month is averaged to form the maximum may be better MODIS synthetic image.

Fujian Province belongs to the coastal areas. In summer, cloudy and even typhoon weather often occurs, which leads to serious loss of data. Luckily, the change has the cycle similarity, so that we can make full use of every 8-days for monthly image integration to constructing monthly LST time series datasets. The MOD11A2 product has more missing values and no available pixels (all defaults to 0 values). A smoothing method is required to maintain the temporal and spatial details by eliminating unusually high or low LST values or null values. Average based smoothing method is applied for this purpose (A monthly LST is probably composed of 3 or 4 remote sensing images). This article takes November 2001 as an example (Figure 1). The LST in November 2001 is calculated by averaging the corresponding 313 days, 321 days and 329 days. Annual datasets are similar to monthly, based on the 12-month average LST replace annual LST (Figure4) (this study take 2001 for example).

\section{RESULTS AND DISCUSSION}

The annual average air temperature trend of Fujian Province is used as the verification data (Table 1) to verify that the datasets constructed in this study is reasonable and reliable. Combined with Table 1, Table 2 and Figure 2 we can see that the LST is higher than the atmospheric temperature, but the two trends are consistent.

\subsection{Temporal Variation Regulation}

From March 2000 to 2015 surface temperature dataset (Table 3 ), we can draw its line chart (Figure 3). We can intuitively learn the inner-annual variation range of LST in 2008 was significantly large than in other years, with the largest above $13.24{ }^{\circ} \mathrm{C}$ in some years. The results also show that the values of LST are relatively violently in most years of 2010 in the past 15 years. The values of LST are highest in summer, lowest in winter, and are similar in spring and autumn, meanwhile, and the values of LST reach their maximum in July and minimum in January or December. There are some extreme points, such as July 2003 LST anomaly high, January 2008 and January 2011 anomaly low. By finding the relevant historical documents, it is found that the year 2003 is the most serious summer and winter drought disaster since 1939, and there is a large-scale continuous high-temperature heat wave in the summer. In 2008, the national strong cooling and snowfall, the winter and winter temperatures in 2011 Continued low, since 1998 since the first warm winter after the first cold winter weather (Change IPOC, 2014).

\subsection{Spatial Variation Regulation}

LST in the spatial distribution of differences is also quite obvious. The LST show distinctive spatial heterogeneity over the whole Fujian province. The results show that the LST of south was higher than of north, and temperature of west is colder than that of east. With the development of society and urbanization, the LST of coastal urban regions was higher than that of rural areas. But there are also a certain number of "heat island" and "cold lake" area (Figure 2). From the two phases $(2001,2015)$ in Figure 2, it can be seen that the relative average annual surface temperature in 2015 is basically the same as that in 2001. The coastal temperature is higher than that of the coastal areas due to the economic development, urbanization and outward expansion in the recent years, especially in Quanzhou and Xiamen city; there is an increasing tendency of LST annually, especially in Fuzhou and Putian city. Meanwhile, high temperature is concentrated in densely populated and economical-commercial areas. In the northwest of Fujian, there was a state of mountainous and forest density, especially in Fujian for the protection of vegetation in recent years, so the 
surface temperature in these areas has been reduced (Changting County). As we know, the LST differed with land use types, and the fitted results were inconsistent between the LST and NDVI. With the increase of NDVI of woodland and grassland, the LST decreased dramatically(Tran et al., 2017), in recent years,
Changting has invested a lot of money and energy in the protection and planting of vegetation, and the vegetation cover has been increased, and the surface temperature of the land has been reduced accordingly.

\begin{tabular}{|c|c|c|c|c|c|c|c|c|c|c|c|c|c|c|c|}
\hline Year & 2001 & 2002 & 2003 & 2004 & 2005 & 2006 & 2007 & 2008 & 2009 & 2010 & 2011 & 2012 & 2013 & 2014 & 2015 \\
\hline Temp & 19.8 & 20.2 & 20.1 & 19.7 & 19.6 & 20.0 & 20.1 & 19.7 & 20.0 & 19.6 & 19.4 & 19.5 & 19.9 & 20.0 & 20.1 \\
\hline
\end{tabular}

Table 1. The annual average air temperature in Fujian Province from 2001 to 2015 year (from the Fujian Provincial Climate Bulletin)

\begin{tabular}{|c|c|c|c|c|c|c|c|c|c|c|c|c|c|c|c|}
\hline Year & 2001 & 2002 & 2003 & 2004 & 2005 & 2006 & 2007 & 2008 & 2009 & 2010 & 2011 & 2012 & 2013 & 2014 & 2015 \\
\hline Temp & 21.9 & 22.4 & 21.9 & 21.9 & 21.6 & 22.1 & 22.2 & 21.6 & 22.4 & 21.7 & 21.0 & 21.5 & 22.2 & 22.0 & 22.0 \\
\hline
\end{tabular}

Table 2. The annual average LST of Fujian Province from 2001 to 2015

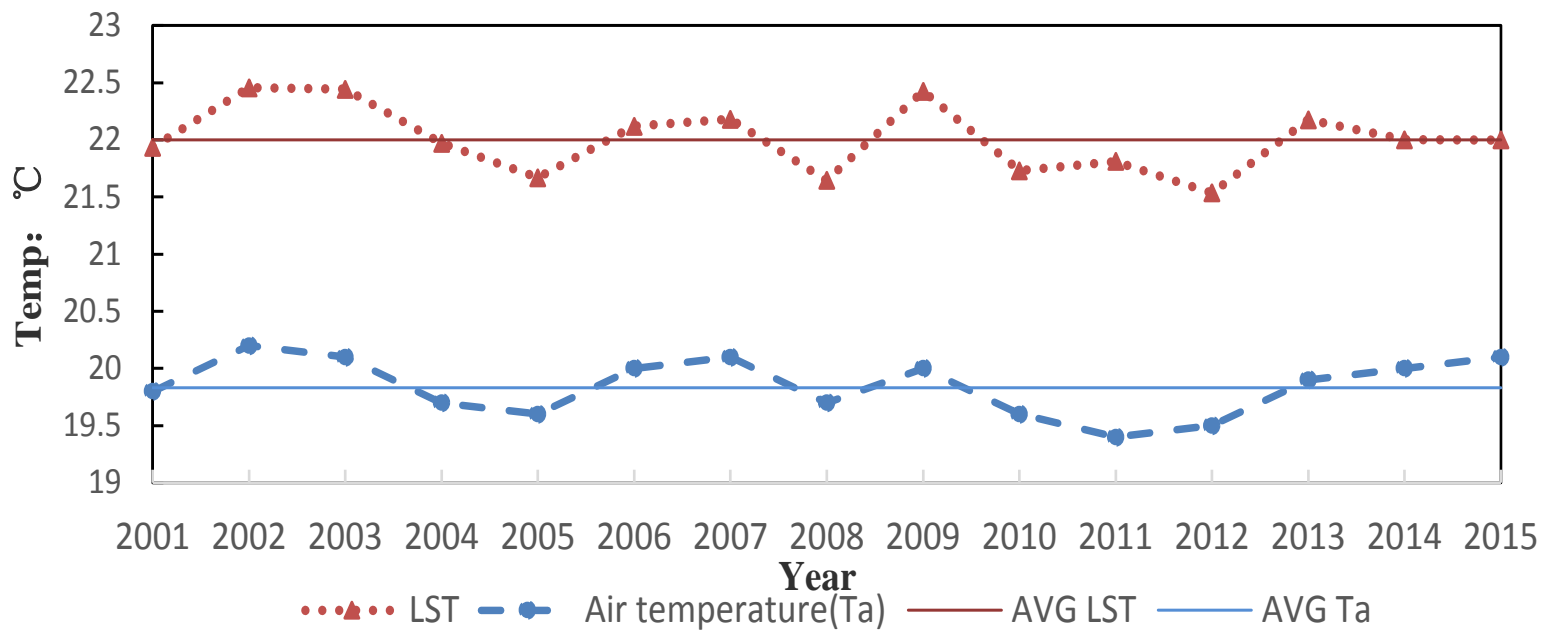

Figure 2. The annual average LST and air temperature of Fujian Province in time series

\begin{tabular}{ccccccccccccc}
\hline Time & Jan & Feb & Mar & Apr & May & Jun & Jul & Aug & Sep & Oct & Nov & Dec \\
\hline 2000 & Null & Null & 21.19 & 23.3 & 27.97 & 27.97 & 28.18 & 26.62 & 26.15 & 22.38 & 19.19 & 16.97 \\
2001 & 14.26 & 18.06 & 21.04 & 21.93 & 26.19 & 27.5 & 27.14 & 26.66 & 23.07 & 24.24 & 18.82 & 14.33 \\
2002 & 14.72 & 17.87 & 21.43 & 25.73 & 24.6 & 27.72 & 28.29 & 26.75 & 25.54 & 22.96 & 18.87 & 15 \\
2003 & 12.3 & 15.98 & 20 & 24.52 & 25.72 & 27.47 & 30.32 & 28.26 & 27.28 & 23.53 & 19.4 & 14.55 \\
2004 & 12.17 & 15.13 & 19.7 & 25.19 & 27.46 & 25.93 & 28.65 & 27.63 & 24.74 & 22.97 & 19.54 & 14.55 \\
2005 & 10.54 & 15.52 & 19.5 & 24.3 & 24.26 & 26.52 & 28.92 & 26.29 & 26.67 & 23.29 & 20.81 & 13.41 \\
2006 & 15.68 & 17.76 & 20.56 & 20.82 & 24.18 & 26.01 & 28.16 & 27.23 & 24.96 & 24.73 & 20.78 & 14.57 \\
2007 & 12.28 & 18.02 & 20.9 & 21.11 & 26.57 & 26.2 & 28.57 & 27.46 & 26.44 & 22.93 & 18.68 & 17 \\
2008 & 9.47 & 13.43 & 21.11 & 25.02 & 25.69 & 26.66 & 29.13 & 27.07 & 26.22 & 24.45 & 17.33 & 14.15 \\
2009 & 13.81 & 21.56 & 19.89 & 23.12 & 26.44 & 26.79 & 27.61 & 26.94 & 26.81 & 24.11 & 19.68 & 12.36 \\
2010 & 15.09 & 20.12 & 19.37 & 19.24 & 26.33 & 25.55 & 28.57 & 27.57 & 25.16 & 21.55 & 18.95 & 13.27 \\
2011 & 9.01 & 16.76 & 18.81 & 25.04 & 27.39 & 27.07 & 27.36 & 28.11 & 25.4 & 23.1 & 10.14 & 13.55 \\
2012 & 14.6 & 17.76 & 19.51 & 22.73 & 24.82 & 26.48 & 27.79 & 26.53 & 24.71 & 23.27 & 17.1 & 13.12 \\
2013 & 13.53 & 17.54 & 21.5 & 22.6 & 25.2 & 27.56 & 29.19 & 27.96 & 26.47 & 23.08 & 19.1 & 12.4 \\
2014 & 14.33 & 16.61 & 19.71 & 24.07 & 24.95 & 26.47 & 27.57 & 26.8 & 26.23 & 24.38 & 19.96 & 12.93 \\
2015 & 14.28 & 16.63 & 22.64 & 23.03 & 24.32 & 26.38 & 27.97 & 27.09 & 24.84 & 23.78 & 20.06 & 12.98 \\
\hline
\end{tabular}




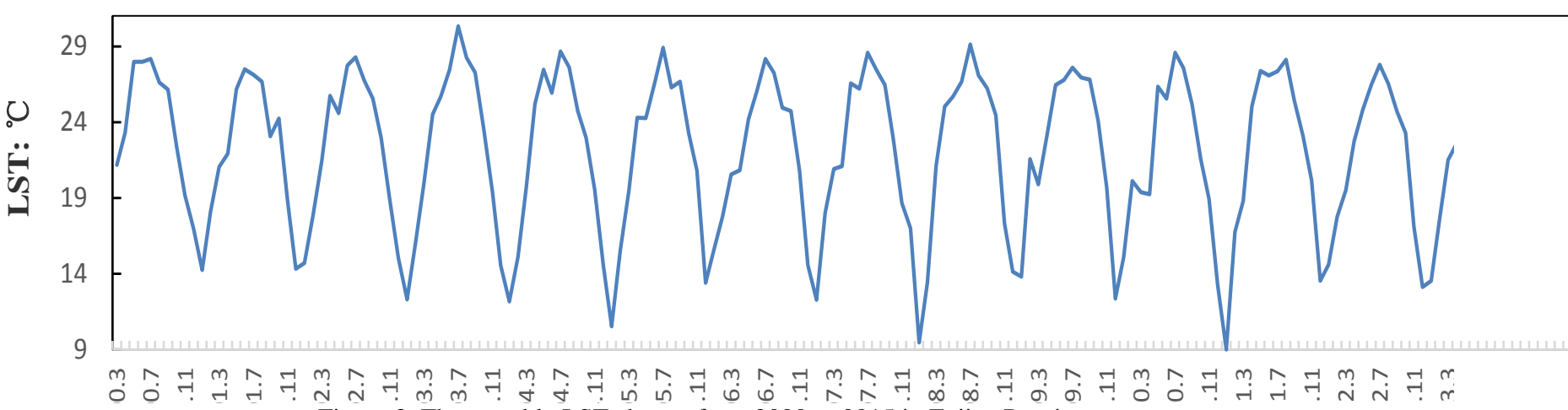

Figure 3. The monthly LST change from 2000 to 2015 in Fujian Province

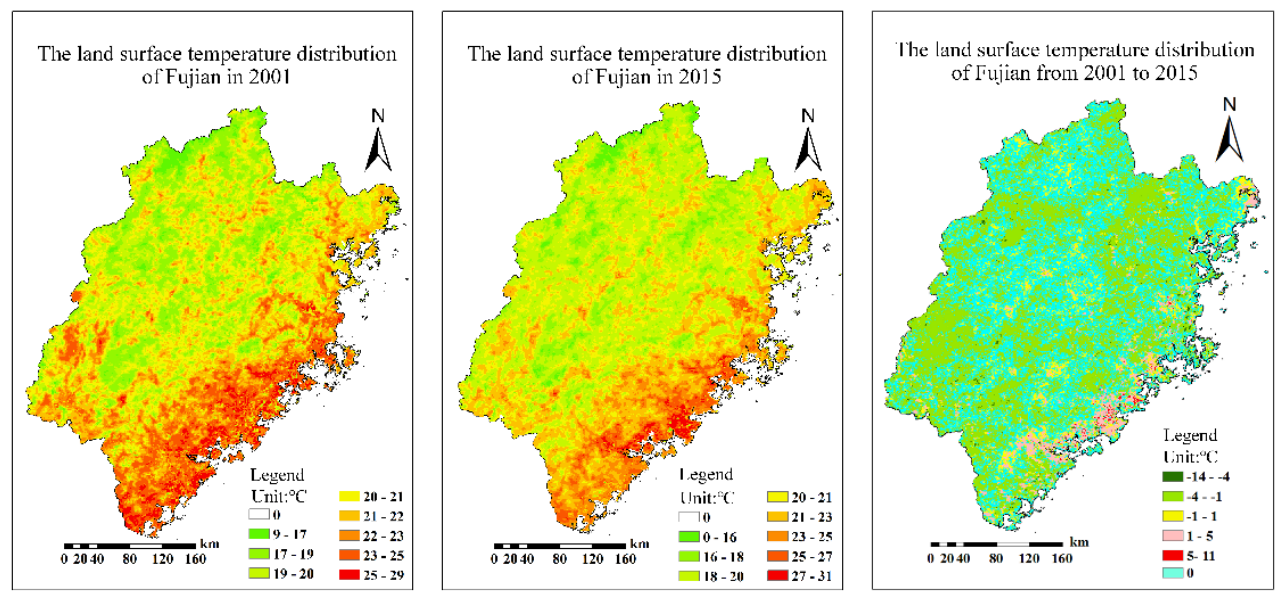

Figure 4. The annual LST spatial distribution in 2001 and 2015 and their differences in Fujian Province

\section{CONCLUTIONS}

Wide range, real-time and cyclical characteristics of the MODIS are fully satisfied with the dynamic monitoring and analysis of the LST. Compared with the traditional way, remote sensing monitoring using MODIS data has its unique and outstanding advantages, which has the ability to macroscopically monitor and analyze about Fujian province, so that in remote areas of research that with mountainous areas become possible.

This paper mainly depends on analyzing the temporal and spatial distribution characteristics of Land surface temperature (LST) in Fujian Province using MODIS/LST time series data from2000 to 2015. The LST of north is colder than that of south, and LST of east is higher than that of west, and the coastal area is higher than that of inland. With the rapid development of urbanization, coastal cities in recent years, the surface temperature reflects an upward trend (especially in Fuzhou, Xiamen, Quanzhou and other coastal areas). However, at the same time Fujian Province makes efforts to protect vegetation, the province's LST showing a downward trend. Through the long-term surface temperature dataset of Fujian Province, the missing data can be supplemented and completed. The LST of Fujian show the sinusoidal curve and fluctuate in the range of $14{ }^{\circ} \mathrm{C} \sim 28{ }^{\circ} \mathrm{C}$. The surface temperature data set which time continuous and space complete has been built, and this paper provides certain theoretical support.

\section{REFERENCES}

Change IPOC, 2014. Climate Change 2014 Synthesis Report. Environmental Policy Collection, 27(2), pp.408.

Ermida S. L., Dacamara C. C., Trigo I F, et al.2017. Modelling directional effects on remotely sensed land surface temperature. Remote Sensing of Environment, 190, pp. 56-69.

Guan Y. L., Wang R. H., Li C., et al. 2015. Spatial-temporal characteristics of land surface temperature in Tianshan Mountains area based on MODIS data. Chinese Journal of Applied Ecology,26(3), pp.681-688.

Pan W. H., Chen J. J., Li W., 2007. Dynamic monitoring of land surface temperature distribution in Fujian province by using remote sensing technology. Chinese Agricultural Science Bulletin, 23, pp. 189-191.

Qin R., 2013. Time series reconstruction of surface temperature based on data assimilation [D]. Central South University.

Quan J., Zhan W., Chen Y., et al. 2016. Time series decomposition of remotely sensed land surface temperature and investigation of trends and seasonal variations in surface urban heat islands. Journal of Geophysical Research Atmospheres, 121(6), pp. 2638-2657.

Tran D X, Pla F, Latorre-Carmona P, et al. 2017. Characterizing the relationship between land use land cover change and land surface temperature. ISPRS Journal of Photogrammetry and Remote Sensing, 124, pp. 119-132. 
Wang X.H., Wang P. X., T. M., et al. 2012. Temporal and spatial distribution characteristics of land surface temperature in Shaanxi province using MODIS data [OL]. Sciencepaper Online ( http://www.paper.edu.cn)

Zhan C. G., Pan W.H., Ji Q., 2011. Dynamic monitoring and spatial-temporal analysis of urban heat island based on MODIS data. Journal of tropical meteorology, 27(3), pp. 396-402. 